\title{
A phase II trial of aclacinomycin-A in advanced squamous cell carcinoma of the head and neck
}

John L. Eckenrode ${ }^{1,2}$, Richard H. Wheeler ${ }^{1, *}$, \& Arlene A. Forastiere ${ }^{1,2}$

${ }^{1}$ Department of Internal Medicine, Division of Hematology/Oncology University of Michigan Hospital Ann Arbor, MI 48109; ${ }^{2}$ Oncology Section, Veterans Administration Medical Center, Ann Arbor, Michigan 48105

Key words: Phase II trial, Aclacinomycin-A, Aclarubicin, Head and Neck Cancer

\section{Summary}

Sixteen patients with advanced squamous cell carcinoma of the head and neck were entered into a phase II trial of Aclacinomycin-A (ACM), $100 \mathrm{mg} / \mathrm{M}^{2}$ administered by brief infusion every three weeks. All patients had received prior radiation therapy and prior non-anthracycline containing chemotherapy. No clinically significant disease regression was observed in fourteen patients having adequate trials. The major toxicity was myelosuppression; leukopenia occurred in $93 \%$ of patients. Gastro-intestinal toxicity was mild and included two patients with transient liver function test abnormalities. No antitumor activity was observed in this patient population which was heavily pre-treated and had a median Karnofsky performance status of only $60 \%$. The results of other phase II trials of ACM-A have been similarly disappointing suggesting that it is not a clinically useful agent in the treatment of solid tumors.

\section{Introduction}

Aclacinomycin-A (ACM-A) is an anthracycline antibiotic, prepared by aerobic fermentation of Streptomyces galilaeus. It was the first of the "Class II" anthracycline antibiotics to enter clinical trials functioning as a more potent inhibitor of RNA synthesis than of DNA synthesis. ACM-A inhibits RNA synthesis at one-tenth the concentration at which it inhibits DNA synthesis. It may intercolate between base pairs of DNA. Cytotoxic effects are partially mediated through generation of free-radical intermediates $(1,2)$. It is cell cycle phase specific and has the maximum cytotoxicity in $\mathrm{L}_{1210}$ cells at the $\mathrm{G}_{1}-\mathrm{S}$ and $\mathrm{S}-\mathrm{G}_{2}$ boundaries.

\footnotetext{
* Present address: Comprehensive Cancer Center, University of Alabama in Birmingham, University Station, Birmingham, Alabama.
}

Preclinical studies in animal and human tumors demonstrated significant activity against $\mathrm{L}_{1210}$ and $\mathrm{P}_{388}$ leukemia cells, lymphosarcoma 6C3 HED cells (4) and human acute myelocytic leukemia (AML) (5). ACM-A in low dose may also act as a differentiating agent for AML cells (6). Phase I trials using a single dose every three weeks determined dose limiting toxicity to be myelosuppression with variable mild hepatic toxicity (6). Cardiac toxicity appeared similar to Adriamycin with respect to acute dysarrhythmic potential causing at least one case of complete heart block which necessitated a transvenous pacemaker (3). Other toxicities were limited to mild nausea and vomiting and to minimal or non-existent alopecia. Tissue necrosis appeared to be much less severe than with Adriamycin (2). A phase II trial of ACM-A in acute lymphocytic leukemia and lymphosarcoma showed significant 
activity but none was observed in other hematologic malignancies (7). Phase II trials in solid tumors have been disappointing with no significant activity observed against bronchogenic carcinoma (8), renal cell carcinoma (9), breast cancer (10) and in one study using a weekly $65 \mathrm{mg} / \mathrm{M}^{2} \mathrm{IV}$ dosage schedule in head and neck cancer (11).

\section{Materials and methods}

Sixteen patients with advanced histologically proven squamous cell cancer of the head and neck region, who were not better treated with surgery or radiation therapy were included. Prior to study entry, all patients had a complete history and physical examination, complete blood count (CBC) with differential, platelet count, blood urea nitrogen, serum creatinine, bilirubin, SGOT, SGPT, LDH, alkaline phosphatase, total serum protein and albumin. A chest radiograph, electrocardiogram and cardiac ejection fraction determination were obtained at the start of therapy and repeated as needed during the course of therapy. Liver-spleen scans, bone scans and computerized tomograms were obtained when clinically indicated. All patients had an expected survival of at least 8 weeks and a Karnofsky performance status of at least $50 \%$. All patients had adequate bone marrow reserve with an initial WBC count greater that $4000 / \mu 1$ and platelet count greater than $150,000 / \mu 1$. Serum bilirubin was less than $2.0 \mathrm{mg} / \mathrm{dl}$ and serum creatinine was less than $2.0 \mathrm{mg} / \mathrm{dl}$. No patient with a recent myocardial infarction or congestive heart failure was eligible.

ACM-A was administered as a 20-30 minute intravenous infusion at an initial dose of $100 \mathrm{mg} / \mathrm{M}^{2}$ repeated every 3 weeks. Dose adjustments were based on nadir counts of the preceding cycle using the following criteria: 1) WBC count greater that 3000/ $\mu \mathrm{l}$ and platelet count greater than $100,000 / \mu 1$, increase the dose $25 \%$; 2) WBC count $2000-3000 / \mu \mathrm{l}$ and platelet count $70-100,000 / \mu \mathrm{l}$, same dose as previous cycle; 3) WBC count $1000-1900 / \mu \mathrm{l}$ or platelet count of $50-75,000 / \mu 1$, decrease the dose $25 \%$; 4) WBC count less than $1000 / \mu \mathrm{l}$ or platelets less than $50,000 / \mu \mathrm{l}$, decrease the dose by $50 \%$. $\mathrm{Pa}$ - tients were followed with weekly CBC and platelet counts and liver function tests. Weight, performance status, and tumor measurements were assessed every three weeks. Cardiotoxicity was to be addressed by resting and exercise MUGA scans before the third dose in all patients in whom a clear response was demonstrated. Therapy was continued until progression of disease was documented.

An adequate trial was defined by the completion of at least one full course of therapy ( 3 weeks) with response and toxicity assessment. Standard criteria were used to define tumor response: complete response (CR) - disappearance of all clinical, $\mathrm{x}$ ray and biochemical evidence of tumor for more than one month; partial response (PR) $-50 \%$ or greater reduction in the sum of the products of the greatest perpendicular diameters of all measurable lesions in the absence of new disease for one month; minor response - between 25 and $49 \%$ reduction in the sum of the products of the greatest perpendicular diameters of all lesions; stabilization - less then $25 \%$ increase in the sum of the products of the greatest diameters of all lesions; disease progression - more than $25 \%$ increase in the sum of the products of the greatest perpendicular diameters of all lesions or the appearance of new lesions.

\section{Results}

Fourteen patients were evaluable for reponse. Two patients died from uncertain causes after the first course of ACM-A before evaluation was complete. Table 1 shows patient characteristics. Median age was 57 and median performance status was 60 . All of the patients had been pre-treated with from one to four different chemotherapy regimens, none of which included Adriamycin. All had received prior full dose radiation therapy and all but two of the evaluable patients had undergone surgical resection. Disease recurred in $64 \%$ of the patients at local sites or in regional neck nodes; $36 \%$ of the patients had disease metastatic to the lung. One patient presented as an unknown primary with midjugular neck nodes. Table 2 details the side effects. Dose limiting toxicity was leukopenia with $93 \%$ of 
Table I. Patient Characteristics.

\begin{tabular}{|c|c|}
\hline \# Evaluable patients & 14 \\
\hline Median age (range) & $57(36-66)$ \\
\hline \multicolumn{2}{|l|}{ Sex: Male 10} \\
\hline \multicolumn{2}{|l|}{ Female 4} \\
\hline \multirow[t]{3}{*}{ Median Performance Status } & $60(50-80)$ \\
\hline & No. of \\
\hline & Patients \\
\hline \multicolumn{2}{|l|}{ Prior Treatment } \\
\hline Surgery & 12 \\
\hline Radiation therapy & 14 \\
\hline Chemotherapy & 14 \\
\hline \multicolumn{2}{|c|}{ No. of Prior Chemotherapy regimens: } \\
\hline 1 & 7 \\
\hline 2 & 6 \\
\hline 3 & 0 \\
\hline 4 & 1 \\
\hline \multicolumn{2}{|l|}{ Primary Sites: } \\
\hline Larynx & 4 \\
\hline Oral Cavity & 2 \\
\hline Oropharynx (base of tongue) & 2 \\
\hline Hypopharynx & 4 \\
\hline Parotid & 1 \\
\hline Unknown & 1 \\
\hline \multicolumn{2}{|l|}{ Sites of measurable disease: } \\
\hline Local-regional & 9 \\
\hline Distant (lung) & 5 \\
\hline
\end{tabular}

Table 2. Toxicity.

\begin{tabular}{ll}
\hline Number of courses of ACM-A & No. of patients \\
$(1)$ & 6 \\
$(2)$ & 5 \\
$(3)$ & 3 \\
Toxicity: & \\
Hematologic nadirs: & \\
WBC $($ Cells $/ \mu$ l) & 5 \\
$2000-3000$ & 6 \\
$1000-1999$ & 0 \\
$<1000$ & \\
PLT $($ Cells $/ \mu 1)$ & 1 \\
$50-100,000$ & 0 \\
$25-49,000$ & 0 \\
$<25,000$ & 2 \\
LFT abnormalities & 5 \\
Nausea/vomiting & 0 \\
Stomatitis & 1 \\
Diarrhea & 0 \\
Alopecia & \\
\hline
\end{tabular}

the patients developing a nadir WBC count below $3000 / \mu 1$ and $43 \%$ below $2000 / \mu \mathrm{l}$. One patient required hospitalization for sepsis while leukopenic. Only mild anemia (hemoglobin drop of less than two grams $\%$ ) and thrombocytopenia were noted. Nausea and vomiting occurred in 5 patients and was grade 3 in 2 patients. Liver function tests, SGOT and alkaline phosphatase, were mildly elevated to less than 2 times normal in 2 patients but resolved with time without stopping therapy. No alopecia or cardiotoxicity was observed. The dose was escalated in 2 patients. There were no dose attenuations. One patient had a stable disease course for two treatments while all other patients rapidly progressed.

\section{Discussion}

In this phase II trial, no clinically significant responses were observed. All patients were heavily pre-treated and although pushed to dose-limiting toxicity, all but one tumor rapidly advanced. The lack of observable antitumor activity may be due to the characteristics of the patient population entered into the trial: median performance status of $60 \%$ and prior exposure to both chemotherapy and radiation therapy in all patients. Our results, however, confirm those reported by Kish et al. (11). We conclude that in this dose and schedule, ACM$A$ is not a useful agent to treat advanced squamous cell carcinoma of the head and neck.

\section{Acknowledgments}

We wish to thank Ms. Wilma Savitski for her excellent secretarial assistance. This trial was supported in part by NCI Grant Contract \# N01-CM07405 .

\section{References}

1. Oki T, Takeuchi T, Oka S. Umezawa H: New anthacycline antibiotic aclacinomycin A: experimental studies and correlation with clinical trials. Rec Res Cancer Res 76:21-40, 1981 
2. Aclacinomycin A: NCI Clinical Brochure, July, 1979

3. Hori S, Shirai M, Hirano S, Oki T, Inni T, Tsukagoshi S, Ishizuka M, Takenchi T, Umezawa $\mathrm{H}$ : Antitumor activity of new anthracycline antibiotics, aclacinomycin-A and its analogs, and their toxicity. Gann 68:685-690, 1977

4. Mathe G, Gescher F, Bayssas M, Misset JL, Machover D, Gilma, Delgado M, Riband P, Hoyat M: Aclacinomycin A in acute leukemia and lymphomas. Lancet: Aug. 11, $310-311,1979$

5. Sakurai M, Sampi K, Hozumi M: Possible differentiation of human acute myeloblastic leukemia cells by daily and intermittent administration of aclacinomycin-A. Leukemia Research 72:139-143, 1983

6. VanEcho DA, Whitacre MY, Aisner J, Applefeld MM, Wiernik PH: Phase I trial of aclacinomycin A. Can Treat Rep 66, 5:1127-1132, 1982

7. Mathe G, Byassas M, Gouveia J, Dantchev D, Riband P, Machover D, Misset JL, Schwarzenberg L, Jasmin C,
Hayat M: Preliminary results of a phase II trial of aclacinomycin in acute leukaemia and lymphosarcoma. Cancer Chemother. Pharmacol. 1, 259-262, 1978

8. Chiuten DF, Carr DT, Dhingra HM, Murphy WK, Spitzer G, Umsawasdi T, Bodey GP, Valdivieso M: AclacinomycinA in metastatic bronchogenic carcinoma. (Abstract) Proc Am Assoc Ca Res. 25:180, 1984

9. Decker DA, Drelichman A, Opipari MI, Al-Sarraf M: Phase II evaluation of aclacinomycin-A in adenocarcinoma of the kidney. Am J Clin Oncol (CCT) 7:527-528, 1984

10. Forastiere AA, Budman DR, Richards F, Aisner J, Weinberg V, Wood WC: Phase II Trial of aclarubicin in advanced breast cancer: a cancer and leukemia group B study. Cancer Treat Rep 67:1137-1138, 1983

11. Kish JA, Al-Sarraf M: Aclacinomycin. Phase II evaluation in advanced squamous cell carcinoma of the head and neck. Am J Clin Oncol (CCT) 7:534-537, 1984 